\title{
First report of human ehrlichiosis in Turkey
}

\author{
Melike Emiroğlu ${ }^{1}$, Bekir Çelebi ${ }^{2}$ \\ ${ }^{1}$ Department of Pediatric Infectious Diseases, Selcuk University Faculty of Medicine, Konya; ${ }^{2}$ Public Health Institution of \\ Turkey, National High Risk Pathogens Reference Laboratory, Ankara, Turkey.E-mail: mkeser17@gmail.com \\ Received: 26th January 2018, Revised: 26th February 2018, Accepted: 3rd March 2018
}

SUMMARY: Emiroğlu M, Çelebi B. First report of human ehrlichiosis in Turkey. Turk J Pediatr 2019; 61: 267-270.

Ehrlichiosis, a tick-borne infection, can cause severe and fatal disease. A 6-year-old boy was admitted with fever, chills, malaise, headache, anorexia, rhinorrhoea, and cough lasting two days. He had had contact with a dog 10 days prior, and a tick had been removed the day before. Fever, minimal conjunctival injection, oropharyngeal hyperemia and cracked, hyperemic lips were observed. Laboratory tests were normal except for lymphopenia and hyponatremia. Cytoplasmic morulae in the monocytes and granulocytes were seen on peripheral blood smear. Doxycycline was started immediately, and the fever subsided within 48 hours. Given the Ehrlichia was positive but Anaplasma negative by real-time PCR, he was diagnosed as ehrlichiosis, subspecies identification could not be performed. This is the first human ehrlichiosis case in Turkey.

Key words: ehrlichiosis, zoonoses, tick-borne diseases, Turkey, morulae.

Tick-borne zoonotic infections due to different microorganisms can cause fatal infections, including rickettsiosis, ehrlichiosis, anaplasmosis and Crimean-Congo haemorrhagic fever (CCHF). ${ }^{1}$ Ehrlichiae are obligate intracellular Gram-negative bacteria that grow in membranebound vacuoles in leukocytes. They can infect humans and other mammals, including dogs, cattle, sheep, goats, rodents, and deer, via tick bite. ${ }^{2}$ Although, CCHF cases have been described in neighboring countries before, the first clinical CCHF cases in Turkey were not recognised until 20023; at present, anyone with a tick bite history and fever is evaluated for CCHF, but not ehrlichiosis. We report a child with ehrlichiosis, representing the first case in Turkey.

\section{Case Report}

In November 2017, a 6-year-old boy from Konya was admitted with fever, chills, malaise, headache, anorexia, rhinorrhoea and cough lasting two days. He had had contact with a street dog 10 days before, and his mother had removed a tick the previous day. The patient's axillary temperature was $39.5^{\circ} \mathrm{C}$, and minimal conjunctival injection, oropharyngeal hyperemia and cracked, hyperemic lips were observed.
The laboratory test results were as follows: white blood cell (WBC) count $11,800 / \mathrm{mm}^{3}$, neutrophils $10,240 / \mathrm{mm}^{3}$ (72.4\%), lymphocytes $600 / \mathrm{mm}^{3}(5.12 \%)$, haemoglobin, $13.1 \mathrm{~g} /$ $\mathrm{dl}$, platelets $236,000 / \mathrm{mm}^{3}$, international normalised ratio (INR) 1.22 , erythrocyte sedimentation rate $7 \mathrm{~mm} / \mathrm{h}, \mathrm{C}$-reactive protein $7.62 \mathrm{mg} / \mathrm{dl}$, procalcitonin, $0.598 \mathrm{ng} /$ $\mathrm{ml}$, serum albumin $4.5 \mathrm{~g} / \mathrm{dl}$, serum sodium $130 \mathrm{mEq} / \mathrm{L}$, aspartate aminotransferase 46 $\mathrm{U} / \mathrm{L}$, alanine aminotransferase $19 \mathrm{U} / \mathrm{L}$, and lactic dehydrogenase $291 \mathrm{U} / \mathrm{L}$. Chest X-ray and echocardiography were normal. Peripheral blood smear was performed, and cytoplasmic morulae were seen in the both of monocytes and granulocytes (Fig. 1.). Doxycycline was started immediately; blood samples were sent to the Public Health Institution of Turkey, National High Risk Pathogens Reference Laboratory.

The patient had diarrhea for one day and intense malaise continued; the WBCs dropped to $3,500 / \mathrm{mm}^{3}$, whereas the lymphocytes rose to $1,620 / \mathrm{mm}^{3}$. Blood, throat and urine cultures were negative. The fever subsided in 24-48 hours. Given that he tested positive for Ehrlichia, but negative for Anaplasma on realtime polymerase chain reaction (PCR) ${ }^{4}$, he was diagnosed with ehrlichiosis. Rickettsiae conorii 


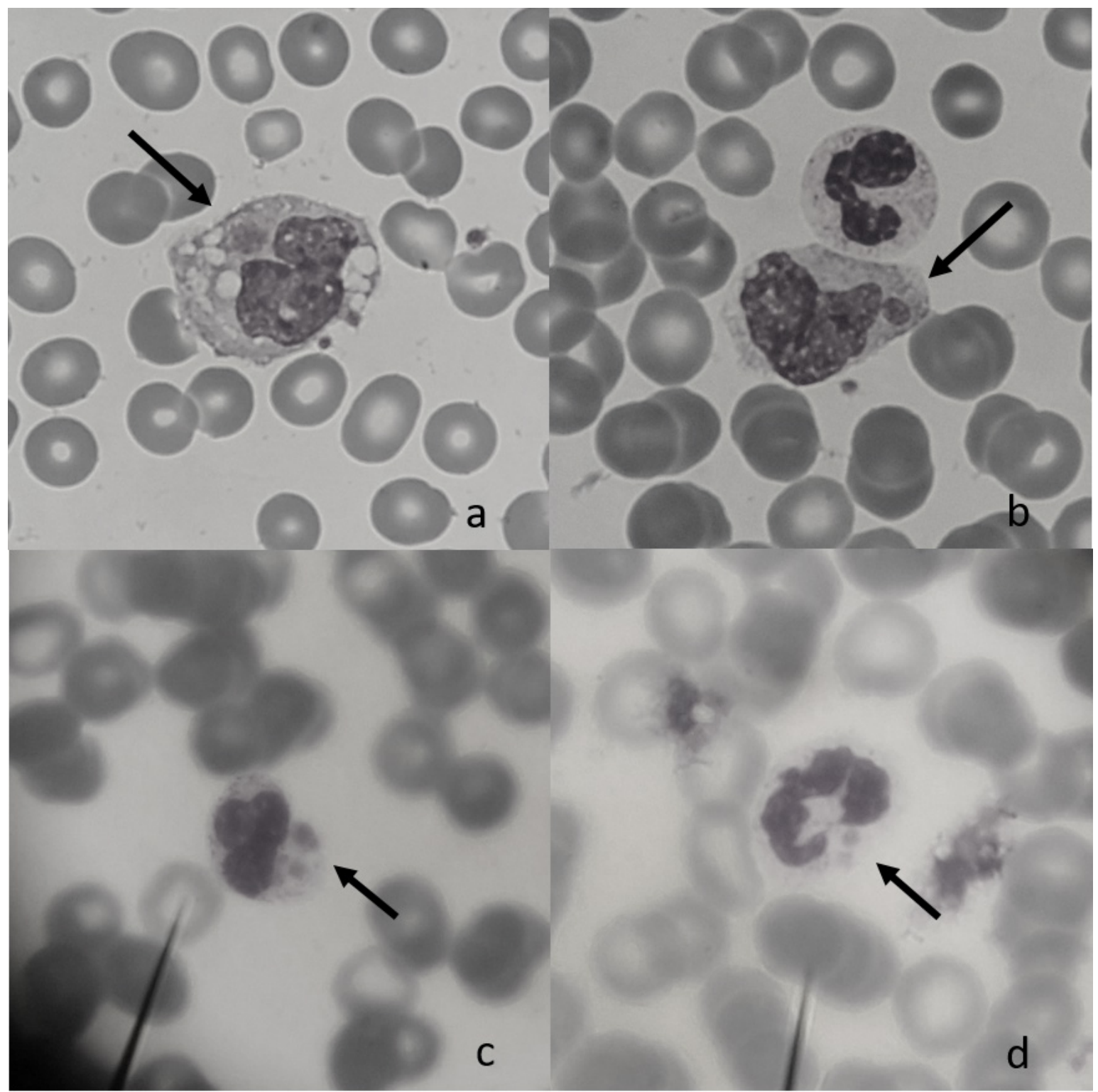

Fig. 1. Peripheral blood $(\times 1000)$. Giemsa staining shows a monocytes $(a, b)$ and granulocytes (c, d) with an intracytoplasmic morulae (arrow).

immunoglobulin (Ig) $\mathrm{M}$ and IgG by enzyme linked immunosorbent assay, and CCHF IgM by indirect immunofluorescence antibody (IFA) and CCHF PCR were also negative. Doxycycline was completed in 10 days.

Informed consent was received from the family.

\section{Discussion}

Ehrlichiosis is an infection caused by bacteria of the Ehrlichia genus in the family Anaplasmataceae, which include E. chaffeensis, E. ewingii, E. canis and E. muris-like agent ${ }^{1}$. E. chaffeensis involves monocytes and macrophages and causes human monocytic ehrlichiosis, whereas E. ewingii involves granulocytes and causes Ehrlichia ewingii ehrlichiosis. These two agents cause most human ehrlichiosis. ${ }^{2}$ A. phagocytophilum, another member of the family Anaplasmataceae, also involves granulocytes, causing human granulocytic anaplasmosis (HGA). ${ }^{1}$

Although it was previously known in animals, the first described cases of human ehrlichiosis involved Ehrlichia chaffeensis and E. ewingii, identified in 1991 in 1999, respectively. ${ }^{2}$ 
Tick-borne diseases are closely related with geographic location of vector ticks. E. chaffeensis and E. ewingii are transmitted to humans by Amblyomma americanum, while A. phagocytophilum comes from Ixodes scapularis. ${ }^{1}$ We were unable to retrieve the dog or tick.

Human ehrlichiosis is increasingly reported in North America and Europe. During 2008-2012, 4,613 cases of E. chaffeensis and, 55 cases of $E$. ewingii infections in humans were reported from United States. ${ }^{2}$ In Europe, the prevalence of the A. phagocytophilum genogroup in I. ricinus ticks is $0.4-66.7 \%$. However, in seroepidemiological studies, the HGA seroprevalence rates were $0-2.9 \%$ in blood donors and $1.5-21 \%$ in tick-exposed individuals. E. ewingii and $E$. chaffeensis cases have not been described. ${ }^{5}$ In Turkey, molecular and serological studies have been evaluated Ehrlichia species in ticks, dogs, ruminates and humans. Christova et al. ${ }^{6}$ found that $5 \%$ of non-Ixodes ticks from Turkey yielded a signal with the Ehrlichia/Anaplasma genus probe on the reverse line blot. The seropositivity rates for $A$. phagocytophilum in I. ricinus ticks was reported as $3.93 \% 7$, while in cattle and sheep it was $10.1-15.3 \%$ and 9.9-13.6\%, respectively. ${ }^{8}$ In cattle, Ehrlichia spp. seropositivity was detected as $1.66 \%^{8}$. Only two studies have evaluated humans for A. phagocytophilum in persons with a history of tick exposure; Ongut et al. ${ }^{9}$ reported a seropositivity of $8 \%$ in Antalya, while Kilic et al. ${ }^{8}$ found a seropositivity of $25 \%$ in the Thrace Region. Unfortunately, no Turkish study has evaluated $A$. americanum, I. scapularis or mammals or humans for E. chaffeensis and E. ewingii seropositivity. We think that the discordance between seropositivity rates in the population and lack of reported ehrlichiosis cases is due to physicians' lack of awareness.

Ehrlichiosis mostly occurs in the months of May to August. The common symptoms are fever, chills, malaise, myalgia, headache, rash, confusion, conjunctival injection and gastrointestinal symptoms like nausea, vomiting, diarrhoea and anorexia. ${ }^{1}$ Rash and gastrointestinal symptoms are less common in E. ewingii infection. Without treatment, symptoms typically resolve after 1-2 weeks, but prompt antimicrobial therapy will shorten the duration and reduce the risk of serious manifestations ${ }^{10}$, such as acute respiratory distress syndrome, encephalopathy, meningitis, disseminated intravascular coagulation, spontaneous hemorrhage and renal failure. ${ }^{1}$ The estimated fatality rate for E. chaffeensis infections is approximately $1-3 \%$, while for anaplasmosis, it is less than $1 \%$; no fatal cases of E. ewingii ehrlichiosis have been reported. ${ }^{10}$ Generally, paediatric patients have a mild infection, but children aged $<10$ years and geriatric or immunosuppressed persons have the highest case-fatality rates ${ }^{1}$. Our patient was admitted in November with flu-like symptoms and tick bite history; global warming may have affected the tick activity.

Characteristic routine laboratory findings for ehrlichiosis are thrombocytopenia, leukopenia, elevated liver enzymes and hyponatremia ${ }^{2}$, similar to anaplasmosis.

After the bacteria infects blood leukocytes, it multiplies in cytoplasmic membranebound vacuoles forming membrane-bound intracytoplasmic bacterial inclusions (morulae, Latin for "mulberry"), which stain dark blue or purple and are stippled in appearance. ${ }^{1}$ Cytoplasmic morulae in the WBCs on peripheral blood smear, bone marrow, or cerebrospinal fluid may indicate infection with Anaplasmataceae; morula positivity in the blood smear is reported in about $17 \%$ of immunocompetent patients and all of immunocompromised hosts. ${ }^{11}$ Interestingly, morulae have not been observed yet in peripheral blood cells in patients with Ehrlichia muris-like agent infection ${ }^{1}$. Although leukocyte tropism has been well described for different ehrlichiae, it is not a strict rule; E. chaffeensis most commonly involves monocytes, while E. ewingii mainly involves granulocytes. ${ }^{10}$ The fourfold increase in the IgG-specific antibody titre by IFA assay between the acute and convalescent period is meaningful. Since $E$. ewingii has never been grown in culture, and no antigens are available for diagnostic use, it must be confirmed via molecular detection. Detection of specific DNA in a clinical specimen by PCR assay is highly specific (60-85\%) and sensitive $(60-85 \%$ for E. chaffeensis infection and $67-90 \%$ for A. phagocytophilum infection) during acute infection. ${ }^{12}$ Although, PCR is the only definitive diagnostic test for E. ewingii infection, the sensitivity and specificity of this test is unknown. ${ }^{12} \mathrm{~A}$ high rate of false positive 
serology usually occurs due to cross reactive antigens shared by Ehrlichia and Anaplasma, but there is no report about false positivity with PCR, but sequence confirmation of the amplified product is necessary to identify infection with certain species. ${ }^{2}$ Blood samples for PCR must be collected before antibiotic therapy is initiated. We collected our patient's blood two days after the doxycycline treatment started; thus, further sequencing for species identification failed. We could not perform IgG-specific antibody titre analysis. Although, identification of morulae in WBC is insensitive, it is highly suggestive of ehrlichiosis and can serve to early diagnosis ${ }^{11}$, as in our patient. It is impossible to distinguish E. chaffeensis infections from E. ewingii based on history, clinical signs and routine tests ${ }^{2}$; in our patient with tick bite history; fever, flu-like symptoms, lymphopenia and mild hyponatremia were present at the admission, we suspected ehrlichiosis because of morulae seen in both of the monocytes and granulocytes cytoplasm and, diagnosed by realtime PCR but, could not be differentiated as E. chaffeensis or E. ewingii infection.

Doxycycline is the drug of choice for human ehrlichiosis and anaplasmosis treatment, regardless of patient age. The recommended paediatric dosage is $4 \mathrm{mg} / \mathrm{kg}$ per day, divided every 12 hours (maximum $100 \mathrm{mg} /$ dose). Treatment should continue for at least 3 days after the fever subsides and until there is evidence of clinical improvement. ${ }^{10}$ If fever persists for $>48$ hours after initiation of therapy, an alternative or additional diagnosis must be considered. ${ }^{1}$ Minimum course of treatment is 5 to 10 days.

In conclusion, patients with a history of tick bite and fever and flu-like symptoms, with variable degrees of anemia, thrombocytopenia, leukopenia and elevated liver enzymes, should be evaluated for tick-borne infections, including ehrlichiosis. We think that the frequency of Ehrlichia infection diagnoses will rise in Turkey with increased awareness of the disease and diagnostic availability.

\section{REFERENCES}

1. Biggs HM, Behravesh CB, Bradley KK, et al. Diagnosis and management of tick-borne rickettsial diseases: Rocky Mountain spotted fever and other spotted fever group rickettsioses, ehrlichioses, and anaplasmosis United States. MMWR Recomm Rep 2016; 65: 1-44.

2. Nichols Heitman K, Dahlgren FS, Drexler NA, Massung $\mathrm{RF}$, Behravesh CB. Increasing incidence of ehrlichiosis in the United States: A summary of National Surveillance of Ehrlichia chaffeensis and Ehrlichia ewingii infections in the United States, 2008-2012. Am J Trop Med Hyg 2016; 94: 52-60.

3. Gozalan A, Esen B, Fitzner J, et al. Crimean-Congo haemorrhagic fever cases in Turkey. Scand J Infect Dis 2007; 39: 332-336.

4. Bell AC, Patela R. A real-time combined polymerase chain reaction assay for the rapid detection and differentiation of Anaplasma phagocytophilum, Ehrlichia chaffeensis, and Ehrlichia ewingii. Diagn Microbiol Infect Dis 2005; 53: 301-306.

5. Blanco JR, Oteo JA. Human granulocytic ehrlichiosis in Europe. Clin Microbiol Infect 2002; 8: 763-772.

6. Christova I, Van De Pol J, Yazar S, Velo E, Schouls L. Identification of Borrelia burgdorferi sensu lato, Anaplasma and Ehrlichia species, and spotted fever group Rickettsiae in ticks from Southeastern Europe. Eur J Clin Microbiol Infect Dis 2003; 22: 535-542.

7. Cetinkaya H, Matur E, Akyazi I, Ekiz EE, Aydin L, Toparlak M. Serological and molecular investigation of Ehrlichia spp. and Anaplasma spp. in ticks and blood of dogs, in the Thrace Region of Turkey. Ticks Tick Borne Dis 2016; 7: 706-714.

8. Kılıç H. Seropositivity of ehrlichiosis in population with tick bite history in rural areas of Thrace region (Thesis). Edirne: Trakya University, Turkey. 2008.

9. Ongut G, Ogunc D, Mutlu G, et al. Seroprevalence of antibodies to Anaplasma phagocytophilum in Antalya, Turkey. Infection 2006; 34: 107-109.

10. American Academy of Pediatrics. Ehrlichia, Anaplasma, and related infections (human ehrlichiosis, anaplasmosis, and related infections). In: Kimberlin DW, Brady MT, Jackson MA, Long SS (eds). Red Book: 2015 Report of the Committee on Infectious Diseases (30th ed). Elk Grove Village, IL: American Academy of Pediatrics; 2015: 329-333.

11. Regunath H, Rojas-Moreno C, Olano JP, Hammer RD, Salzer W. Early diagnosis of Ehrlichia ewingii infection in a lung transplant recipient by peripheral blood smear. Transpl Infect Dis 2017; 19: e12652

12. Dumler JS, Madigan JE, Pusterla N, Bakken JS Ehrlichioses in humans: epidemiology, clinical presentation, diagnosis, and treatment. Clin Infect Dis 2007; 45(Suppl 1) : S45-S51. 